\title{
Calcific constrictive pericarditis due to rheumatoid arthritis
}

\author{
Hadi Manji and Peter Raven
}

Department of Medicine, St. James' Hospital, Balham, London SW12, UK.

\begin{abstract}
Summary: A patient with calcific constrictive pericarditis due to rheumatoid arthritis is presented: the literature reveals only one previous case which was attributed to the long duration of the rheumatoid arthritis.
\end{abstract}

\section{Introduction}

While pericarditis is a common feature of rheumatoid arthritis, haemodynamically significant constriction is rare and a review of the literature revealed only one previous case of a 74 year old man with arthritis for 20 years, in whom pericardial calcification was attributed to the long duration of rheumatoid pericarditis. ${ }^{1}$ Our patient had symptoms of rheumatoid arthritis for only 4 years.

\section{Case report}

A 34 year old man was referred because of pitting oedema of the legs, lasting one year and unresponsive to diuretic treatment. $\mathrm{He}$ also gave a 4-year history of joint pains affecting the knees, elbows and small joints of the hands. A diagnosis of rheumatoid arthritis had been made 2 years previously when his rheumatoid titre was 1 in 160, but he was lost to follow up and never received treatment.

On examination he was cachectic with mild proximal interphalangeal joint swelling in the hands and rheumatoid nodules on both elbows. There was a BCG scar over the left deltoid. The pulse was 90 per minute and regular, and the blood pressure $100 / 80 \mathrm{mmHg}$ with no pulsus paradoxus. The jugular venous pressure was raised to $10 \mathrm{~cm}$ with prominent ' $a$ ' and ' $v$ ' waves and a positive Kussmaul's sign. Auscultation of the heart revealed a loud third heart sound. There was pitting oedema of the legs to mid-calf. Abdominal examination revealed moderate hepatosplenomegaly.

Correspondence: H. Manji, M.A., M.R.C.P., Rita Lila Weston Institute for Neurological Studies, Middlesex Hospital, Mortimer Street, London W1, UK.

Accepted: 26 May 1989
Laboratory investigations showed a raised erythrocyte sedimentation rate of $35 \mathrm{~mm} / \mathrm{h}$, and abnormal liver function tests (bilirubin $28 \mu \mathrm{mol} / \mathrm{l}$, alkaline phosphatase $208 \mathrm{IU} / \mathrm{l}$, albumin $38 \mathrm{~g} / \mathrm{l}$, $\gamma$-glutamyl transferase $105 \mathrm{IU} / \mathrm{l}$, alanine aminotransferase $16 \mathrm{IU} / \mathrm{l}$ ). Plasma urea and serum calcium and phosphate were normal. The rheumatoid (RAHA) titre was strongly positive at 1 in 5120 , but other autoantibodies were negative. A Mantoux test was negative at 1 in 1000 . The electrocardiogram showed sinus rhythm with evidence of left and right atrial enlargement, and the chest radiograph showed extensive pericardial calcification (Figure 1). On echocardiography the left ventricle showed no late diastolic filling, consistent with constriction. Cardiac catheterization revealed the end diastolic pressure to be similar in all four chambers, with sharp ' $x$ ' and ' $y$ ' descents in the right atrial trace confirming constrictive pericarditis.

At operation the pericardium was found to be thickened and heavily calcified, requiring extensive resection. Histological examination of the resected pericardial calcification. Tuberculosis was excluded in this case by the negative Mantoux test and histological examination of the excised pericardium.

\section{Discussion}

Tuberculosis remains a common cause of constrictive pericarditis, ${ }^{2}$ and is associated with pericardial calcification. Tuberculosis was excluded in this case by the negative Mantoux test and histological examination of the excised pericardium.

Fibrous pericarditis is the commonest cardiac complication of rheumatoid arthritis, an association which was first described by Charcot in $1881^{3}$ : autopsy studies suggest the overall incidence may 


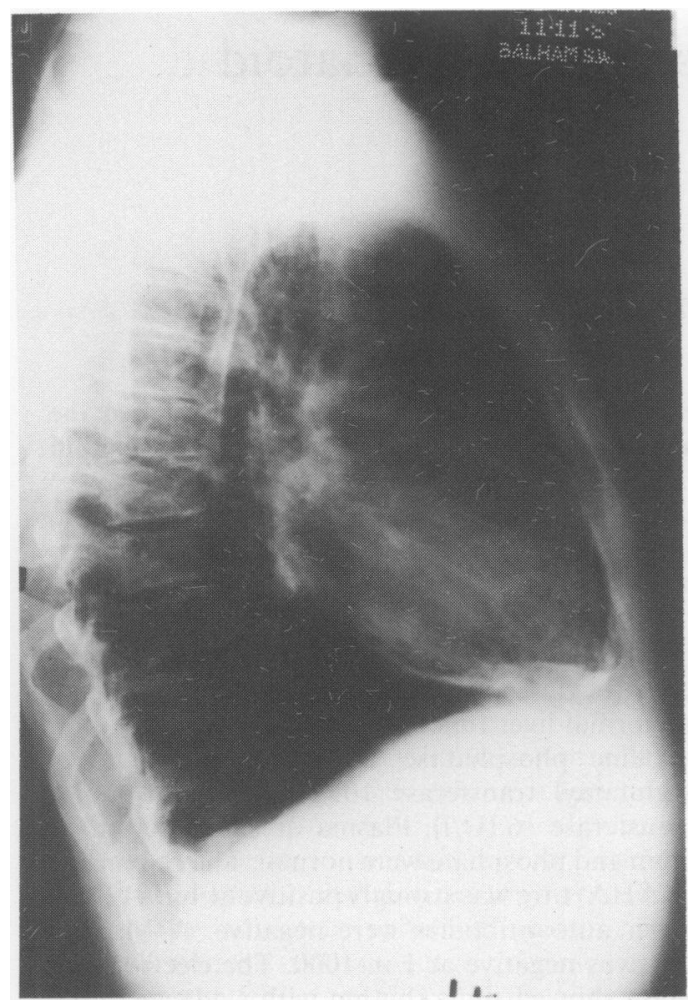

Figure 1 Lateral chest radiograph showing extensive pericardial calcification.

\section{References}

1. Arthur, A., Oskvig, R. \& Basta, L.L. Calcific rheumatoid constrictive pericarditis with cardiac failure treated by pericardiectomy. Chest 1973, 64: 769-771.

2. Anonymous editorial. Rheumatoid constrictive pericarditis. Br Med J 1979, 2: 755.

3. Charcot, J.M. Clinical Lectures on Senile and Chronic Diseases. The New Sydenham Society, London, 1881, 95: pp. 172-175. be as high as $30-50 \% .^{4}$ The much rarer condition of constrictive rheumatoid pericarditis is associated with the male sex, presence of rheumatoid nodules and high rheumatoid factor titres, ${ }^{5}$ all features of the case we present.

The one previously reported case of calcific constrictive pericarditis due to rheumatoid arthritis was in a 74 year old man who had had arthritis for 24 years, and the calcification was attributed to the duration of pericarditis.' In the case we present, a 34 year old man with symptoms of arthritis for only 4 years and no history of pericarditis before presentation, it seems most unlikely that the duration of pericardial disease was a factor in producing calcification. It is of note that a routine chest X-ray in 1977 showed no pericardial calcification.

Like other reported cases of constrictive rheumatoid pericarditis he has made a good recovery following pericardiectomy and his joint symptoms have abated with non-steroidal anti-inflammatory drug treatment.

\section{Acknowledgements}

We would like to thank Dr Bateman for allowing us to report this case and Mrs Gill Porter for typing the manuscript.

4. Kirk, J. \& Cosh, J. The pericarditis of rheumatoid arthritis. $Q J$ Med 1969, 38: 397-423.

5. John, T.J., Hough, A. \& Sergent, J.S. Pericardial disease in rheumatoid arthritis. Am J Med 1979, 66: 385-390. 